\title{
Spring-Plate Resonator Sensor for Measuring Viscosity of Small Amounts of Complex Fluids
}

\author{
Erwin K. Reichel ${ }^{1 *}$, Jan Vermant ${ }^{2}$, Bernhard Jakoby ${ }^{3}$, Christine E.A. Kirschhock ${ }^{1^{*}}$ \\ ${ }^{1}$ Centre for Surface Chemistry and Catalysis, KU Leuven, Leuven, Belgium \\ ${ }^{2}$ Department of Chemical Engineering, KU Leuven, Leuven, Belgium \\ ${ }^{3}$ Institute for Microelectronics and Microsensors, Johannes Kepler University Linz, Austria \\ *) corresponding authors' emails: erwin.reichel@biw.kuleuven.be, christine.kirschhock@biw.kuleuven.be
}

\section{Introduction}

Viscosity and density are physical properties of fluids that are critical for manifold processes in industry. Beside their direct importance of determining the flow characteristics, they can also be used to quantify the chemical condition, e.g., of lubricants, fuels, electrolytes, or the progress of crystallization or polymerization. Viscometers and densitometers are commonly used for measuring these material properties to a high accuracy.

While density is a well-defined property, viscosity is revealed only during dynamic (shear) deformation and may depend on the way it is actually measured. This is specifically true for materials known as non-Newtonian and viscoelastic fluids - where the reaction force depends on the rate, amount, frequency, or duration of the deformation. Often these effects are accompanied by an elastic behavior, so the respective dynamic properties are the viscoelastic storage and loss moduli denoted as $G^{\prime}(\omega)$ and $G "(\omega)$, respectively.

At small deformation, the response is assumed to be linear, so a complex material response function $G^{*}=G^{\prime}+i G^{\prime \prime}$ can be defined for shear as well as for compression, $E^{*}=E^{\prime}+i E^{\prime \prime}$. Another common notation is the complex viscosity, related to the shear modulus by $\eta^{*}=G^{*} /(i \omega)$. Knowing the full spectral behavior not only is required for calculating the response to arbitrary deformation like wave propagation, but also allows insight in relaxation and diffusion processes at the molecular scale.

Since there is no single instrument or sensor that could cover the whole range, the goal is to design devices that measure at frequencies of particular interest. We aim at frequencies one to two orders of magnitude above what can be accessed by laboratory rheometers (typically $100 \mathrm{~Hz}$ ), and at sensors with online capability.

\section{Sensor Principle}

Electromechanical resonators are sensitive to the parameters of the surrounding medium and therefore can be used as sensors for viscoelastic properties and density. In the literature, a variety of concepts are present, e.g. piezoelectric bulk and surface guided wave devices, cantilever-like structures, and MEMS resonators, which are used to measure the viscosity and mass density of (in most cases) Newtonian liquids [1]. Quartz shear-mode resonators have been used for viscoelastic media, but the high frequency range represents a significantly different rheometric regime [2]. In our recent work, we presented a metallic plate resonator excited by Lorentz forces in a permanent magnetic field [3].

While previously, the resonator was fully immersed in the sample liquid, in this contribution we will study the response if the sensor is exposed partly. Basically there are two modes of operation. In one case, a droplet is deposited on the round surface as shown in Fig. 1. Alternatively, a reflector is placed parallel to the resonator plane to form a filled gap - the sample liquid is maintained in place by its surface tension. Under certain conditions, reflected shear waves travelling in the defined gap width extend the viscosity range as well as the capability to measure at frequencies where standing-wave resonances occur. 


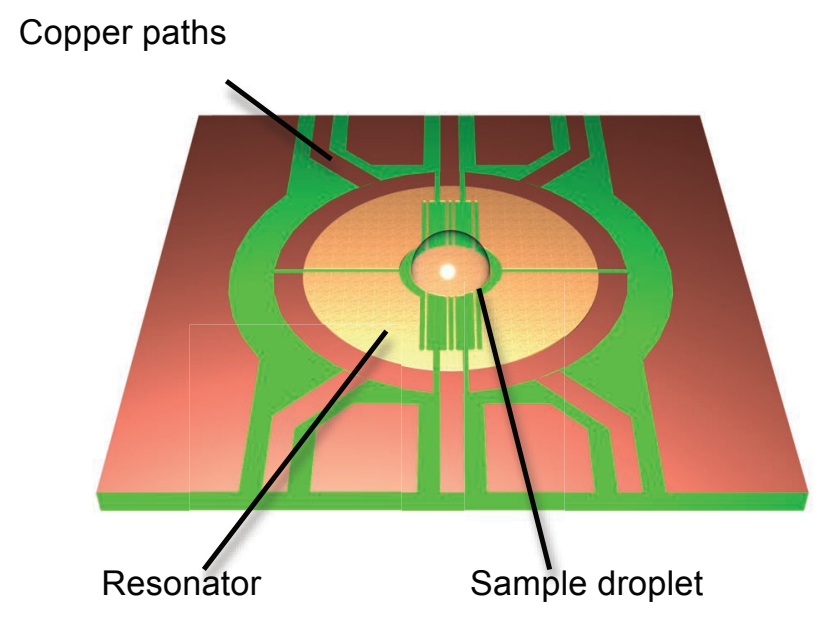

Figure 1: Schematic of the sensor element, the metallic sensor element is mounted directly on the PCB copper structure on a FR4 substrate.

\section{Theory and Numerical Analysis}

Although the resonator is a distributed system, the sensor response is described as a function of a few parameters only, which requires a reduction in the modeling order. Resonators are commonly modeled by decomposition in eigenmodes. The displacement field is written as a series,

$$
\boldsymbol{u}(\boldsymbol{r}, t) \approx \sum_{i=1}^{N} q_{i}(t) \boldsymbol{U}_{i}(\boldsymbol{r}),
$$

where $q_{i}(t)$ are the generalized coordinates of motion and $\boldsymbol{U}_{i}(\boldsymbol{r})$ are the solutions of the mechanical equations in the computational domain with respective boundary conditions (free or fixed). Figure 2 shows a numerical solution obtained using finite elements analysis (COMSOL 4.1). For each eigenmode, the frequency response resembles the behavior of a lumped oscillator (second order system). The electromechanical coupling allows resonance modeling in the electrical domain. Figure 3 shows the electrical equivalent circuit. The main challenge of the sensor modeling is to derive a relation between the resonance parameters and the fluid properties to be measured.

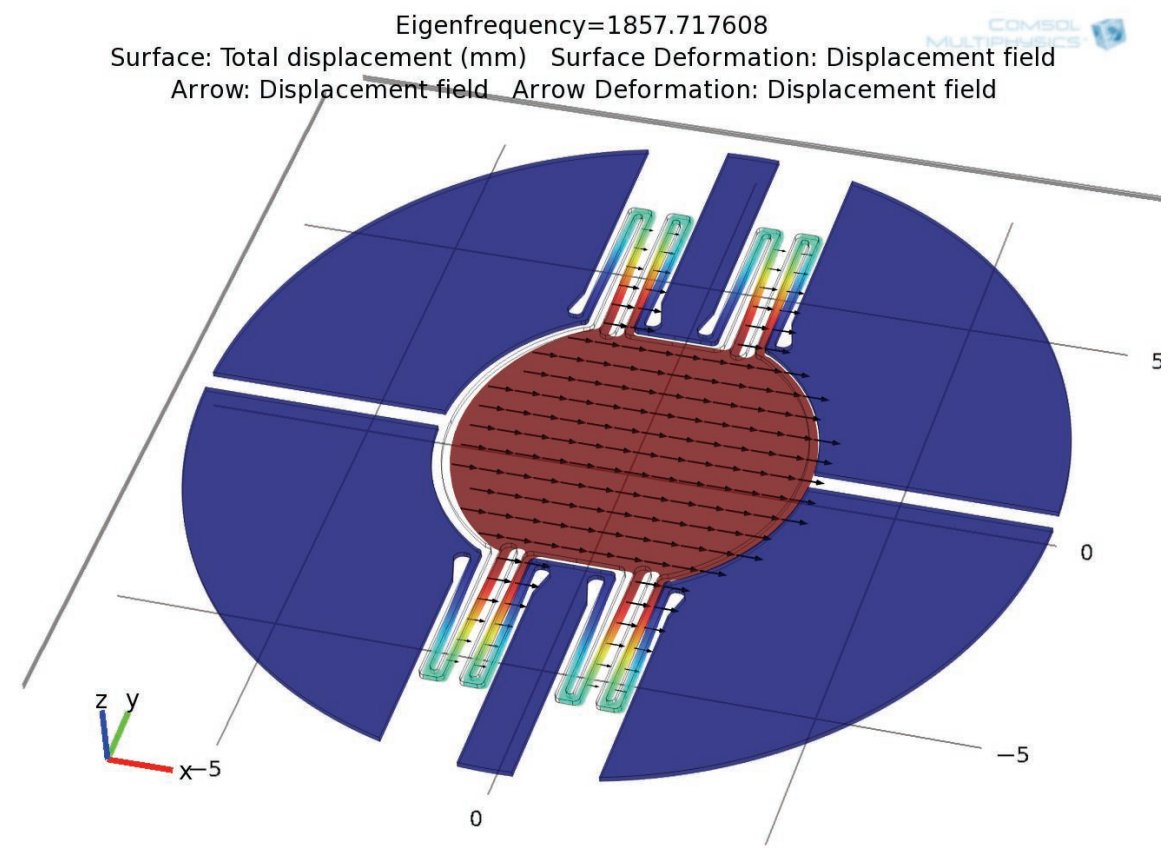

Figure 2: FEA of in-plane eigenmode at $1858 \mathrm{~Hz}$ (undamped) using COMSOL 4.1 structural mechanics module - deformation is scaled up for visibility. 


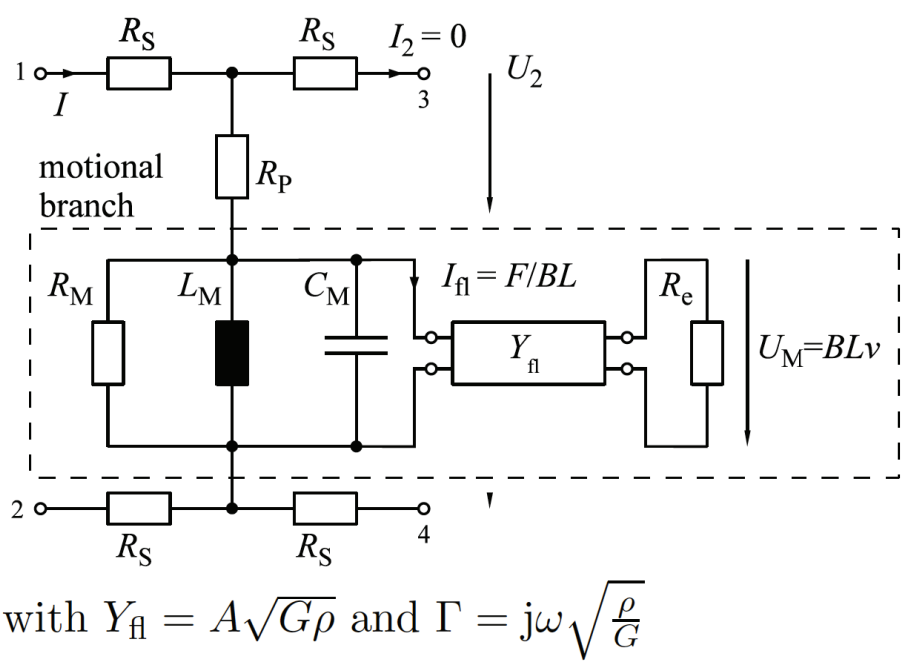

Figure 3: Electrical equivalent circuit model incorporating the reflected shear wave interaction by a transmission line model. $C_{M}, L_{M}$, and $R_{M}$ represent the mass, mechanical stiffness, and intrinsic damping, respectively, $\Gamma$ is the transmission coefficient, $Y_{f l}$ the characteristic acoustic shear impedance, $G=G^{*}$ is the complex (frequency dependent) shear modulus of the sample fluid. I is the excitation current, $U_{\mathrm{M}}$ is the motion-induced voltage, and $U_{2}$ is the measured readout voltage.

In the case of the in-plane eigenmode shown in Fig. 2 the plane shear wave solution is a reasonable approximation in order to derive standing wave solutions in a transmission line model. The shear wave damping in Newtonian liquids is high. Shear waves can only propagate significant distances in elastic and viscoelastic media. In cases where the viscoelastic loss angle is low enough, and the gap width is in the same order of magnitude as the penetration depth, standing wave resonances can be obtained. Such a case is depicted in Fig. 4, where the velocity amplitude along the gap distance is shown over the frequency [4].

For resonator modes exhibiting out-of-plane motion, the fluid displacement can be solved by a potential flow and a correction of the tangential components by a shear wave solution (assuming incompressibility) [5].

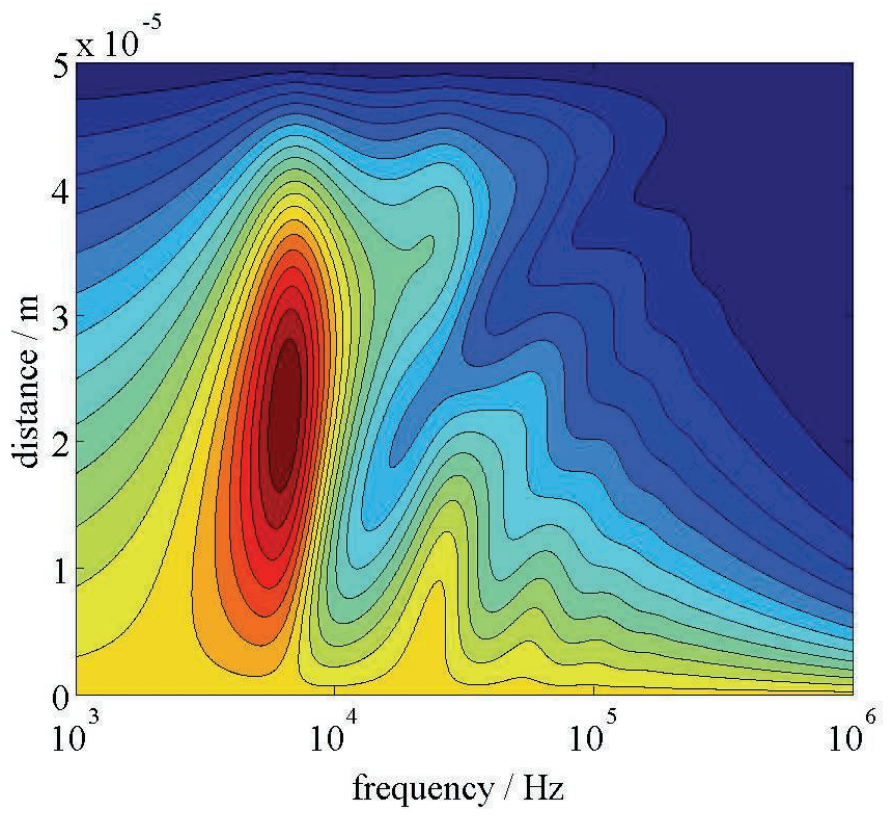

Figure 4: Calculated shear velocity in a $50 \mu \mathrm{m}$ gap for a viscoelastic material ( $\eta=11 \mathrm{mPa}$.s, loss angle $0.23 \pi)$, plotted over the frequency. The appearance of multiple resonances (standing wave modes) provides a novel way to measure the high frequency viscoelastic parameters. 


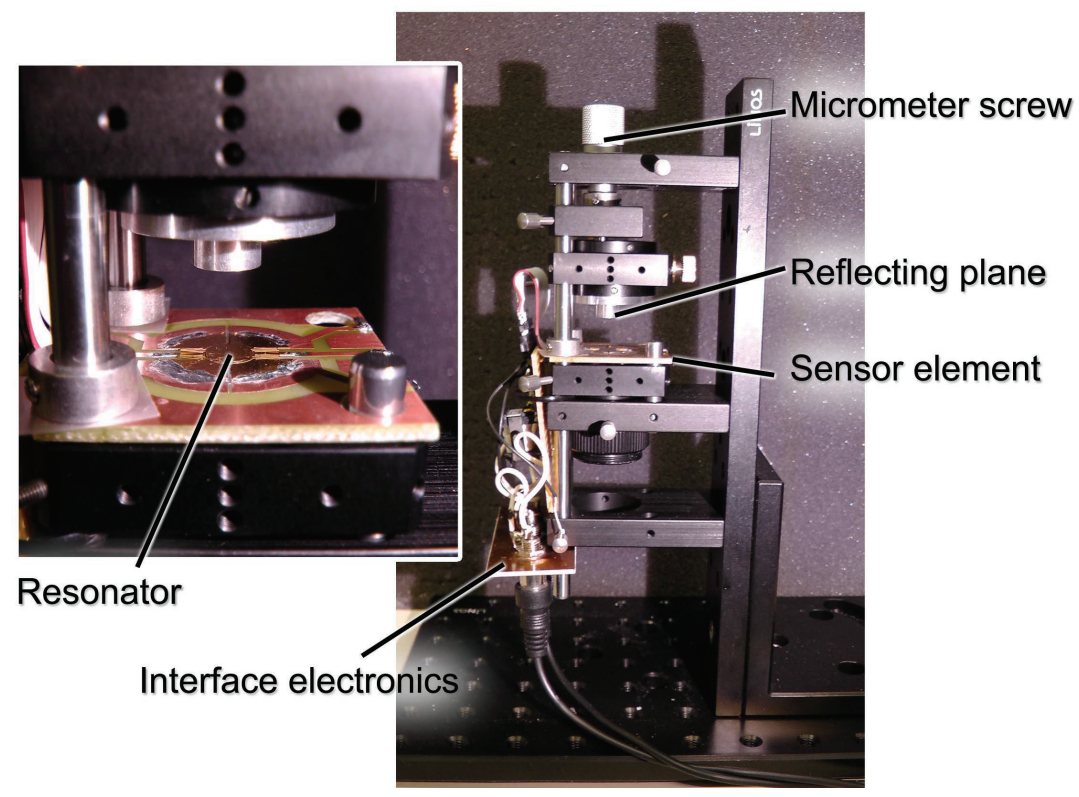

Figure 5: Photograph of the measurement setup, the gap to the reflector can be positioned accurately by a micrometer screw.

\section{Experiments and Results}

The resonator element was integrated in a mechanically stable setup shown in Fig. 5. Putting a droplet on the circular plate performs loading of the sample. In the case of aqueous solutions with respective high surface tension, the amount used was 50 microliters. Optionally, a reflector parallel to the resonator can be lowered using a micrometer screw (this feature was not used for the measurements performed).

Close to the sensor, signal transformers are mounted which connect to an audio interface. This way, sufficient sensitivity is obtained to measure the frequency response [6]. The system responds linearly, so a high-bandwidth signal can be used. Typical measurements take about four seconds, where the voltage is recorded with a sample rate of $48 \mathrm{kHz}$. The frequency response is obtained by deconvolution in the Fourier domain. The resonance characteristics are calculated by first subtracting the electric response (obtained without magnetic field), and then fitting a second order system.

Measurements were performed in a temperature stabilized environment $\left(24.5^{\circ} \mathrm{C}\right)$ using the sample liquids shown in Table I. The Brookfield viscosity standards are based on silicone oil. Using the audio interface, the signal-to-noise-ratio limits the maximum measurable viscosity to about $500 \mathrm{mPa}$.s. The method is most sensitive in the low viscosity range, as is demonstrated with the $30 \mathrm{wt} \%$ silica nanosuspension (LUDOX®) diluted with various amounts of water to yield the given volume fraction $\Phi$. The suspension viscosity was estimated using the Maron-Pierce model with parameters from [7]. Differences in viscosity of $0.2 \mathrm{mPa}$.s can clearly be distinguished.

Table I: List of sample liquids, shear viscosity, density, calculated penetration depth, and measured resonance frequency and quality factor

\begin{tabular}{|c|c|c|c|c|c|c|}
\hline $\begin{array}{l}\mathrm{N} \\
\mathrm{r} .\end{array}$ & Description & $\begin{array}{c}\eta \\
\text { mPa.s }\end{array}$ & $\begin{array}{c}\rho \\
\mathrm{kg} \cdot \mathrm{m}^{-3}\end{array}$ & $\begin{array}{c}\delta \\
\mu \mathrm{m}\end{array}$ & $\begin{array}{c}f_{r} \\
H z\end{array}$ & $\mathbf{Q}$ \\
\hline 1 & Brookfield 5 & 4.9 & 760 & 37.1 & 1492.9 & 49.9 \\
\hline 2 & Brookfield 50 & 49 & 760 & 118.2 & 1469.8 & 19.5 \\
\hline 3 & Brookfield 500 & 490 & 760 & 384.3 & 1389.5 & 9.3 \\
\hline 4 & Water & 0.9 & 998 & 13.7 & 1495.7 & 69.8 \\
\hline 5 & $\Phi=2.9 \%$ silica in $\mathrm{H}_{2} \mathrm{O}$ & 1.1 & 1044 & 14.7 & 1494.7 & 64.5 \\
\hline 6 & $\Phi=5.8 \%$ silica in $\mathrm{H}_{2} \mathrm{O}$ & 1.3 & 1088 & 15.9 & 1493.7 & 59.7 \\
\hline 7 & $\Phi=8.8 \%$ silica $\AA$ in $\mathrm{H}_{2} \mathrm{O}$ & 1.6 & 1132 & 17.5 & 1490.2 & 55.0 \\
\hline 8 & $\Phi=11.7 \%$ silica in $\mathrm{H}_{2} \mathrm{O}$ & 2.1 & 1176 & 19.4 & 1488.2 & 49.0 \\
\hline 9 & $\Phi=14.6 \%$ silica in $\mathrm{H}_{2} \mathrm{O}$ & 2.7 & 1220 & 21.9 & 1485.9 & 45.3 \\
\hline
\end{tabular}


The resonance frequency of the resonator operated in air is $1518 \mathrm{~Hz}$, showing a quality factor of 334.3. The difference to the simulated eigenfrequency of $1858 \mathrm{~Hz}$ is mainly attributed to geometrical deviations caused by the lithography/etching process - uncertainty in material parameters and limitations of the mesh were excluded to a large extent.
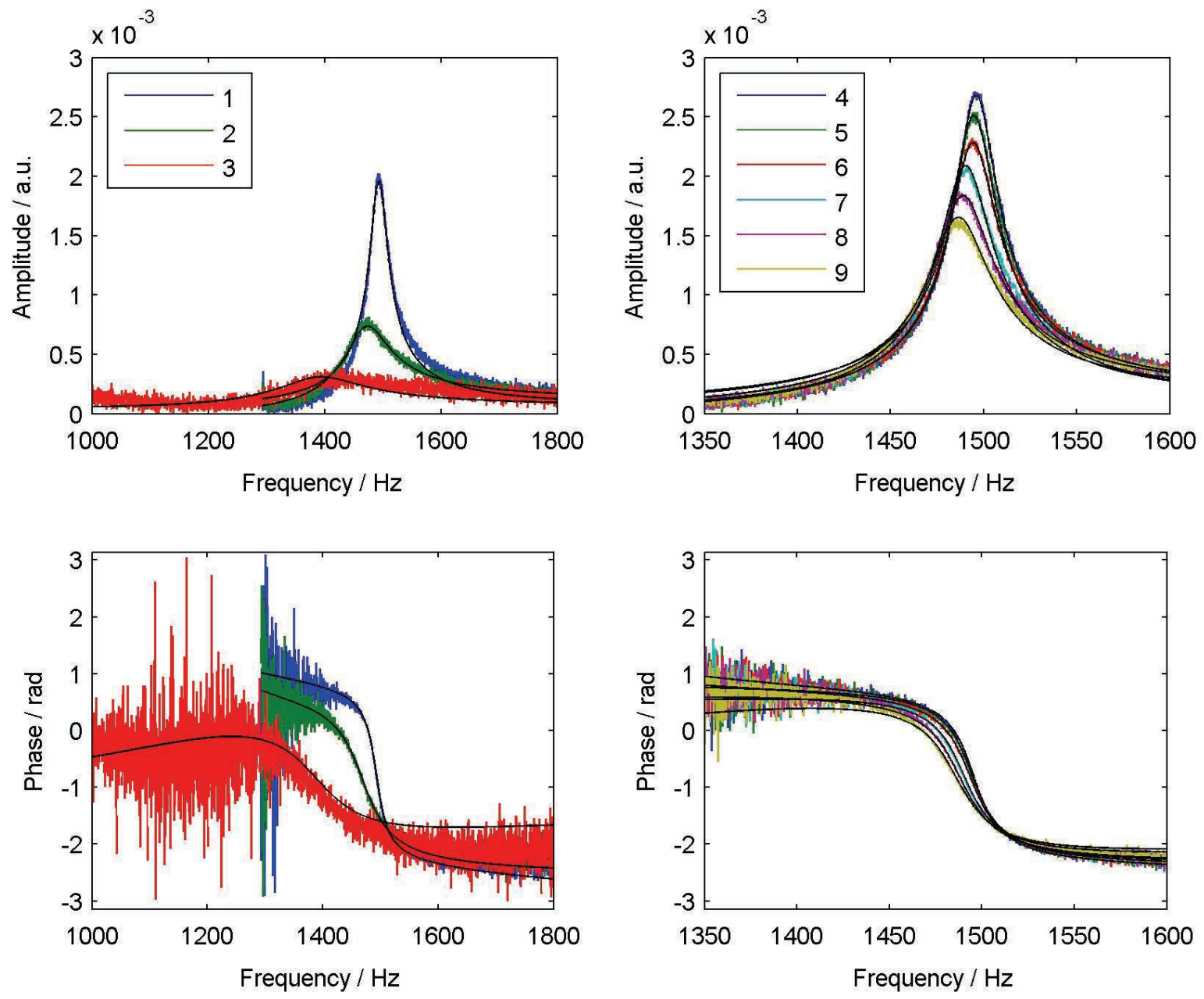

Fig. 6: The measured frequency responses are shown for the viscosity standards (samples 1-3), and for the diluted aqueous silica suspension (samples 4-9).

\section{Conclusions}

With appropriate sensor design and modeling methods, it is in the best case possible to measure the dynamic storage and loss moduli as well as the density of fluids exhibiting complex rheological behavior. The deformation is usually small (displacements in the range of micrometers) so that the response is linear.

We present a resonator sensor for this purpose with the ability to exert almost pure shear deformation to a small (maximum of $50 \mu \mathrm{l}$ ) sample volume and featuring an accurate yet inexpensive measurement method using an audio interface. A potential application is the measurement of concentration or the detection of solvent evaporation of colloidal suspensions, as was demonstrated using a silica nanosuspension.

The aim of the ongoing research is to achieve better theoretical understanding, establish novel sensor principles, and use state-of-the-art fabrication techniques to increase the range of applications for online measurement of rheological properties. A focus is set to technologies that allow mass production, e.g. lithography, polymer processing techniques or silicon micromachining at wafer scale. 


\section{Acknowledgements}

E.K.R. and C.E.A.K. acknowledge financial support by ESA and the Belgian Prodex office (project AO2004-027). The work was supported by the Belgian government through the IAP-PAI network and by the Flemish government through long term structural funding to J.A.M. (Methusalem).

\section{References}

[1] B. Jakoby, R. Beigelbeck, F. Keplinger, F. Lucklum, A. Niedermayer, E.K. Reichel, C. Riesch,T. Voglhuber-Brunnmaier, B. Weiss, Miniaturized Sensors for the Viscosity and Density of Liquids Performance and Issues, IEEE Trans. UFFC, 57/1, 2010, pp 111-120

[2] R. Lucklum, C. Behling, R.W. Cernosek, S.J. Martin, Determination of complex shear modulus with thickness shear mode resonators, J. Phys. D: Appl . Phys, Vol 30 Nr 3, 1997

[3] E.K. Reichel, C. Riesch, F. Keplinger, C.E.A. Kirschhock, B. Jakoby, Analysis and experimental verification of a metallic suspended plate resonator for viscosity sensing, Sensors and Actuators A: Physical, 162/2, 2010, pp 418-424

[4] J. L. Schrag, Deviation of Velocity Gradient Profiles from the "Gap Loading" and "Surface Loading" Limits in Dynamic Simple Shear Experiments, Trans. Soc. Rheol. 21/3, 399, 1977; doi:10.1122/1.549445

[5] E.K. Reichel, C. Riesch, F. Keplinger, B. Jakoby, Modeling the fluid-structure interaction in a fluidic sensor cell, Sensors and Actuators A: Physical, 156/1 2009, pp 222-228

[6] F. Lucklum, E. K. Reichel, B. Jakoby, Miniature flow-through resonator cell for density and viscosity sensing, Procedia Engineering, Volume 5, Eurosensor XXIV Conference, Eurosensor XXIV Conference, 2010, Pages 1413-1417, ISSN 1877-7058, doi: 10.1016/j.proeng.2010.09.380.

[7] C. Riesch, A. Jachimowicz F. Keplinger, E.K. Reichel, B. Jakoby, A micromachined doublyclamped beam rheometer for the measurement of viscosity and concentration of silicon-dioxide-inwater suspensions, Sensors, 2008 IEEE, pp.391-394, 26-29 Oct. 2008, doi: 10.1109/ICSENS.2008.4716461 